\title{
Demyelinating Diseases of the CNS (Brain and Spine)
}

\author{
Frederik Barkhof and Kelly K. Koeller
}

\section{Learning Objectives}

- To be familiar with the differential diagnosis of white matter diseases

- Understand the importance of clinical setting, mode of presentation and lab results in idiopathic inflammatory disorders

- Be able to apply MS and NMO diagnostic criteria

- Appreciate the value of spinal cord imaging in the work-up of MS

- Recognize the most important variants and mimics of MS

\section{Key Points}

- Age-related white matter changes are extremely prevalent and affect the deep white matter with sparing of the U-fibers and spinal cord

- The McDonald criteria for MS include cortico/juxtacortical, periventricular, infratentorial and spinal cord lesions

- Longitudinally extensive spinal cord lesions and large/atypical brain lesions should prompt antibody testing to rule out NMOSD

- The differential diagnosis of symmetric white lesions includes toxic and metabolic disorders

F. Barkhof $(\bowtie)$

Department of Radiology and Nuclear Medicine, VU University

Medical Center, Amsterdam, The Netherlands

UCL Institutes of Neurology and Healthcare Engineering,

London, UK

e-mail: f.barkhof@vumc.nl

\section{K. K. Koeller}

Section of Neuroradiology, Department of Radiology,

Mayo Clinic, Rochester, MN, USA

\subsection{Introduction}

Demyelinating disorders of the central nervous system (CNS) that affect the brain and spine have a variety of etiologies and can be separated into primary such as multiple sclerosis (MS) and other idiopathic inflammatory-demyelinating diseases (IIDDs) and secondary (e.g., infectious, ischemic, metabolic, or toxic) diseases. MRI is the imaging modality of choice to assess demyelinating disorders of the brain and the cord and, together with the clinical and laboratory findings, can accurately classify them in most cases [1-3]. This review will highlight the important imaging manifestations of some acquired demyelinating diseases that allow more specific diagnosis.

\subsection{Idiopathic Inflammatory- Demyelinating Diseases (IIDDs) of the CNS}

The term IIDD encompasses a broad spectrum of CNS disorders that can be differentiated according to their severity, clinical course, and lesion distribution, as well as their imaging, laboratory, and pathological findings. The spectrum includes monophasic, multiphasic, and progressive disorders, ranging from highly localized forms to multifocal or diffuse variants [2]. Relapsing-remitting (RR) and secondary progressive (SP) MS are the two most common forms of IIDD. MS can also have a progressive course from onset (primary progressive [PP]). Fulminant forms of IIDD include a variety of disorders that have in common the severity of the clinical symptoms, an acute clinical course, and atypical findings on MRI. The classic fulminant IIDD is Marburg disease but is extremely rare. Baló's concentric sclerosis and acute disseminated encephalomyelitis (ADEM) can also present with severe, acute attacks.

Some IIDDs have a restricted topographic distribution, as is the case with neuromyelitis optica spectrum disorders (NMOSD), which can have a monophasic, but more often follows a relapsing course. 


\subsection{Multiple Sclerosis}

MS is a progressive inflammatory, demyelinating and neurodegenerative autoimmune disease characterized pathologically by perivascular infiltrates of mononuclear inflammatory cells, demyelination, and axonal loss and gliosis, with the formation of focal and diffuse abnormalities in the brain and spinal cord, mainly affecting the optic nerves, brainstem, spinal cord, and cerebellar and periventricular white matter, although cortical and subcortical gray matter damage is also prominent, resulting in chronic progressive disability for the majority of people with the disorder.

The high sensitivity of MRI in depicting brain and spinal cord demyelinating plaques has made this technique the most important paraclinical tool in current use, not only for the early and accurate diagnosis of MS, but also for understanding the natural history of the disease and monitoring and predicting the efficacy of disease-modifying treatments [4].

MRI is the most sensitive imaging technique for detecting MS plaques throughout the brain and spinal cord. Proton density (PD) or T2-weighted MR images (especially acquired using the fluid-attenuated inversion recovery (FLAIR) sequence) show areas of high signal intensity in the periventricular white matter in $>90 \%$ of MS patients (the remainder may have cord lesions only). MS plaques are generally round to ovoid in shape and range from a few millimeters to more than $1 \mathrm{~cm}$ in diameter. They are typically discrete and focal at the early stages of the disease, but become confluent as the disease progresses, particularly in the posterior hemispheric periventricular white matter. MS plaques tend to affect the periventricular and juxtacortical white matter, whereas small vessel ischemic lesions tend to involve the deep white matter more [4]. The total $\mathrm{T} 2$ lesion volume of the brain increases by approximately 5-10\% each year in the relapsing forms of MS.

Both acute and chronic MS plaques appear hyperintense on T2/FLAIR sequences, reflecting their increased tissue water content. The signal increase indicates edema, inflammation, demyelination, reactive gliosis, and/or axonal loss in proportions that differ from lesion to lesion. The vast majority of MS patients have at least one ovoid periventricular lesion, whose major axis is oriented perpendicular to the outer surface of the lateral ventricles. The ovoid shape and perpendicular orientation derive from the perivenular location of the demyelinating plaques noted on histopathology (Dawson's fingers).

MS lesions tend to affect specific regions of the brain, including the periventricular white matter, the inferior surface of the corpus callosum, the cortico-juxtacortical regions, the temporal lobes, and the infratentorial regions. Focal involvement of the periventricular white matter in the anterior temporal lobes is typical for MS and rarely seen in other white matter disorders, especially not in aging/hypertension (see table below). The lesions commonly found at the calloso-septal interface are best depicted by sagittal
T2-FLAIR images-a sequence highly recommended for diagnostic MRI studies.

Characteristic differences between small-vessel disease (SVD) and MS

\begin{tabular}{|l|l|l|}
\hline Involvement & SVD & MS \\
\hline Corpus callosum & Rare & Common \\
\hline U-fibers & Rare & Often \\
\hline Brainstem & Central pons & Peripheral \\
\hline Temporal lobe & Never $^{\mathrm{a}}$ & Often \\
\hline $\begin{array}{l}\text { Gadolinium } \\
\text { enhancement }\end{array}$ & $\begin{array}{l}\text { Exceptional } \\
\text { (subacute infarction) }\end{array}$ & Common \\
\hline Black holes & Rare & Typical \\
\hline Lacunes & Typical & Never \\
\hline Spinal cord & Never & Common \\
\hline
\end{tabular}

${ }^{a}$ With the exception of cerebral autosomal dominant arteriopathy with subcortical infarcts and leukoencephalopathy (CADASIL)

Histopathological studies have shown that a substantial portion of the total brain lesion load in MS is located within the cerebral cortex. Presently available MRI techniques are not optimal for detecting cortical lesions because of poor contrast resolution between normal-appearing gray matter (NAGM) and the plaques in question, and because of the partial volume effects of the subarachnoid spaces and CSF surrounding the cortex. Cortical lesions are better visualized by 2D or 3D FLAIR sequences and newer MR techniques such as 3D double inversion recovery (DIR) MR sequences which selectively suppress the signal from white matter and cerebrospinal fluid (CSF). Juxtacortical lesions that involve the "U" fibers are seen in two-thirds of patients with MS.

Posterior fossa lesions preferentially involve the floor of the fourth ventricle, the middle cerebellar peduncles, and the brainstem. Most brainstem lesions are contiguous with the cisternal or ventricular CSF spaces, and range from large confluent patches to solitary, well-delineated paramedian lesions or discrete "linings" of the CSF border zones. Predilection for these areas is a key feature that helps to identify MS plaques and to differentiate them from focal areas of ischemic demyelination and infarction that preferentially involve the central pontine white matter.

Approximately $10-20 \%$ of $\mathrm{T} 2$ hyperintensities are also visible on T1-weighted images as areas of markedly decreased signal intensity compared with gray matter. These so-called T1 black holes have a different pathological substrate that depends, in part, on the lesion age. Temporary hypointensity is present in up to $80 \%$ of recently formed lesions and probably represents marked edema, with or without myelin destruction or axonal loss. In most cases, the acute lesions become isointense within a few months as inflammatory activity abates, edema resolves, and reparative mechanisms like remyelination become active. Less than $40 \%$ evolve into persisting or chronic "black holes" [5], which correlate pathologically with the most severe demye- 
lination and axonal loss, indicating areas of irreversible tissue damage. Chronic black holes are more frequent in patients with progressive disease than in those with RRMS disease, and more frequent in the supratentorial white matter as compared with the infratentorial white matter. They are rarely found in the spinal cord and optic nerves.

MS lesions of the spinal cord resemble those in the brain. The lesions can be focal (single or multiple) or diffuse, and mainly affect the cervical cord segment. On sagittal scans, the lesions characteristically have a cigar shape and rarely exceed two vertebral segments in length (the so-called shortsegment lesions in contrast to longitudinally extensive lesions in NMO). On cross-section, they typically occupy the lateral and posterior white-matter columns, border the surface but may extend to involve the central gray matter, and rarely occupy more than one half the cross-sectional area of the spinal cord [6].

Acute spinal cord lesions can produce a mild to moderate mass effect with some spinal cord swelling and may show contrast enhancement. Active lesions are rarer in the spinal cord than the brain and are more frequently associated with new clinical symptoms. The prevalence of spinal cord abnormalities is as high as 74-92\% in established MS, and depends on the clinical phenotype of MS. Asymptomatic spinal cord lesions are found in 30-40\% of patients with a clinically isolated syndrome (CIS), even if the presenting symptoms do not involve the spinal cord clinically. In RRMS, the spinal cord lesions are typically multifocal. In secondary progressive MS, the abnormalities are more extensive and diffuse and are commonly associated with spinal cord atrophy. In primary progressive MS, spinal cord abnormalities are quite extensive as compared with brain abnormalities. This discrepancy may help to diagnose primary progressive MS in patients with few or no brain abnormalities.

Longitudinal and cross-sectional MR studies have shown that the formation of new MS plaques is often associated with contrast enhancement, mainly in the acute and relapsing stages of the disease [7]. The gadolinium enhancement varies in size and shape, usually lasting a few weeks, although steroid treatment shortens this period. Incomplete ring enhancement on T1-weighted gadolinium-enhanced images with the open border facing the gray matter of the cortex or basal ganglia is a common finding in active MS plaques and is a helpful feature for distinguishing between inflammatory-demyelinating lesions and other focal lesions such as tumors or abscesses which will have a closed ring of enhancement [8].

Contrast enhancement is a relatively good predictor of further enhancement and of subsequent accumulation of T2 lesions, but shows no (or weak) correlation with progression of disability and development of brain atrophy. In RRMS and early SPMS, enhancement is more frequent during relapses and correlates well with clinical activity. For patients with primary progressive MS, serial T2-weighted studies show few new lesions and less frequent enhancement.
Contrast-enhanced T1-weighted images are routinely used in the study of MS to provide a measure of inflammatory activity in vivo.

MRI-based disease activity is 5-10 times more frequently than clinical evaluation of relapses, suggesting that most of the enhancing lesions are clinically silent. Subclinical disease activity with contrast-enhancing lesions is 4-10 times less frequent in the spinal cord than the brain, a fact that may be partially explained by the large volume of brain as compared with spinal cord. High doses of gadolinium and a long post-injection delay can increase the detection of active spinal cord lesions.

The diagnosis of MS relies heavily on MRI information, certainly when there is a clinically isolated syndrome suggestive of demyelination. MRI can be used to show dissemination in space (lesions 2 of 4 locations - juxtacortical, periventricular, infratentorial or spinal cord) and to demonstrate dissemination in time (enhancement or new $\mathrm{t} 2$ lesions at follow-up).

\subsection{Multiple Sclerosis Variants}

\subsubsection{Baló's Concentric Sclerosis}

Baló's concentric sclerosis is a rare IIDD subtype, considered a variant of MS, with characteristic radiologic and pathologic features. The disease was formerly considered an aggressive MS variant, leading to death in weeks to months after onset, and in which the diagnosis was made on histopathologic findings at postmortem examination. However, with the widespread use of MRI, this MS variant is often identified in patients who later have a complete or almost complete clinical recovery [9]. The pathologic hallmarks of are large demyelinated lesions showing a peculiar pattern of alternating layers of preserved and destroyed myelin. One possible explanation for this pattern is that sublethal tissue injury is induced at the edge of the expanding lesion, which would then stimulate expression of neuroprotective proteins to protect the rim of peri-plaque tissue from damage, thereby resulting in alternating layers of preserved and non-preserved myelinated tissue [10]. These alternating bands are best identified with T2-weighted sequences, which typically show thick concentric hyperintense bands corresponding to areas of demyelination and gliosis, alternating with thin isointense bands corresponding to normal myelinated white matter. This pattern can be also identified on T1-weighted images as alternating isointense (preserve myelin) and hypointense (demyelinated) concentric rings. These bands, which may eventually disappear over time, can appear as multiple concentric layers (onion-skin lesion), as a mosaic, or as a "floral" configuration. The center of the lesion usually shows no layering because of massive demyelination ("storm center"). Restricted diffusion followed by contrast enhance- 
ment is common in the outer rings (inflammatory edge) of the lesion.

The Baló pattern on MRI can be isolated, multiple, or combined with typical MS-like lesions, and the lesion structure can vary from one or two to several alternating bands, with a total size from one to several centimeters. Lesions occur predominantly in the cerebral white matter, although brainstem, cerebellum, and spinal cord involvement has also been reported [11].

\subsubsection{Neuromyelitis Optica Spectrum Disorders}

Neuromyelitis optica (NMO) is an autoimmune inflammatory disorder of the CNS with a predilection for the optic nerves and spinal cord [12]. The discovery of an NMOspecific autoantibody directed against aquaporin-4 (AQP4-Ab), the major water channel in the CNS, clearly identified $\mathrm{NMO}$ as a disease separate from $\mathrm{MS}$ - and requiring a different treatment than MS [13, 14].

This uncommon and topographically restricted form of IIDD is characterized by severe unilateral or bilateral optic neuritis and complete transverse myelitis, which occur simultaneously or sequentially over a varying period (weeks or years). The index events of new-onset NMO are severe unilateral or bilateral optic neuritis, acute myelitis, or a combination of these symptoms. The myelitis attacks appear as complete transverse myelitis with severe bilateral motor deficits, sensory-level, bowel and bladder dysfunction, pain and significant residual neurologic injury. Optic neuritis attacks are generally more severe than those typically seen in MS.

Approximately $85 \%$ of patients have a relapsing course with severe acute exacerbations and poor recovery, which leads to increasing neurologic impairment and a high risk of respiratory failure and death due to cervical myelitis. Patients who experience acute optic neuritis and transverse myelitis simultaneously or within days of each other are much more likely to have a monophasic course. On the other hand, a relapsing course correlates with NMO-IgG seropositivity, a longer interval between attacks, older age at onset, female gender, and less severe motor impairment after the myelitic onset. Although the initial attacks are more severe in patients proven to have monophasic NMO, the long-term neurologic prognosis is somewhat better in this group because patients do not accumulate disability from recurrent attacks.

Clinical features alone are insufficient to diagnose NMO; CSF analysis and MRI are usually required to confidently exclude other disorders. CSF pleocytosis ( $>50$ leukocytes/ $\mathrm{mm}^{3}$ ) is often present, while oligoclonal bands are seen less frequently (20-40\%) than in MS patients (80-90\%). AQP4-Ab detection is best performed using cell-based assays that have greater sensitivity. AQP4-Ab distinguish this form of IIDD from MS and it can predict relapse and conversion to NMO in patients presenting with a single attack of longitudinally extensive myelitis. NMO-IgG is positive in $52 \%$ of patients with relapsing transverse myelitis and in $25 \%$ of patients with recurrent idiopathic optic neuritis [15].

Wingerchuk et al. [16] proposed a revised set of criteria for diagnosing NMO. These criteria remove the absolute restriction on CNS involvement beyond the optic nerves and spinal cord, allow any interval between the first events of optic neuritis and transverse myelitis, and emphasize the specificity of longitudinally extensive spinal cord lesions on MRI and NMO-IgG-seropositive status. More recently the International Panel for NMO Diagnosis developed new diagnostic criteria that define the unifying term NMOSD, which is stratified by serologic testing (with or without AQP4-IgG) [17]. These new criteria require, in patients with AQP4-IgG, core clinical and MRI findings related to optic nerve, spinal cord, area postrema, other brainstem, diencephalic, or cerebral presentations. However, more stringent clinical and MRI criteria are required for diagnosis of NMO-spectrum disorders without AQP4-IgG or when serologic testing is unavailable.

\section{Diagnostic Criteria for NMOSD Without or Unknown AQP4-IgG Status}

1. At least two core clinical characteristics occurring as a result of one or more clinical attacks and meeting all of the following requirements:

a. At least one core clinical characteristic must be optic neuritis, acute myelitis with LETM, or area postrema syndrome

b. Dissemination in space (two or more different core clinical characteristics)

c. Fulfillment of additional MRI requirements, as applicable (see below)

2. Negative tests for AQP4-IgG using best available detection method or testing unavailable

3. Exclusion of alternative diagnoses

\section{Additional MRI Requirements}

1. Acute optic neuritis: requires brain MRI showing

a. Normal findings or only nonspecific white matter lesions

b. Optic nerve MRI with T2w-hyperintense lesion or T1-weighted gadolinium-enhancing lesion extending over $>1 / 2$ optic nerve length or involving optic chiasm

2. Acute myelitis: requires associated intramedullary MRI lesion extending over $\geq 3$ contiguous segments (LETM) or $\geq 3$ contiguous segments of focal spinal cord atrophy in patients with history compatible with acute myelitis 
3. Area postrema syndrome: requires associated dorsal medulla/area postrema lesions

4. Acute brainstem syndrome: requires associated periependymal brainstem lesions

MRI of the affected optic nerve demonstrates swelling and loss of blood-brain barrier integrity with gadolinium enhancement that can extend into the optic chiasm. The spinal cord lesions in NMO typically extend over three or more contiguous vertebral segments and occasionally the entire spinal cord (longitudinally extensive spinal cord lesions); they are centrally located (preferential central gray-matter involvement) and affect much of the cross-section on axial images. During the acute and subacute phase, the lesions are tumefactive and show contrast uptake. In some cases, the spinal cord lesions are small at the onset of symptoms, mimicking those of MS, and then progress in extent over time. The presence of very hyperintense spotty lesions on T2-weighted images ("bright spotty sign") is a specific feature that helps differentiate NMO from MS, particularly in patients without longitudinally extensive spinal cord lesions [6, 11, 12], and likely reflects the highly destructive component of the inflammatory lesion. Spinal cord lesions can progress to atrophy and necrosis and may lead to syrinx-like cavities on T1-weighted images.

NMO was long considered a disease without brain involvement, and a negative brain MRI at disease onset was considered a major supportive criterion for the diagnosis of NMO. However, various studies have shown that brain MRI abnormalities exist in a significant proportion (50-85\%) of patients [18]. Brain MRI lesions are often asymptomatic, but sometimes are associated with symptoms even at disease onset. The brain lesions are commonly nonspecific. They can be dot-like or patchy, $<3 \mathrm{~cm}$ in diameter, and located in the deep white matter, brainstem, or cerebellum. Nonetheless, some brain MRI features appear to be quite characteristic and distinct from MS lesions. These abnormalities may parallel sites with high AQP4 expression adjacent to the ventricular system at any level, such as the hypothalamus, periependymal areas surrounding the third and lateral ventricles, cerebral aqueduct, corpus callosum, and dorsal brainstem adjacent to the fourth ventricle. The appearance of periventricular lesions in NMO is quite characteristic. In contrast to MS, where periventricular lesions are discrete, oval-shaped, and perpendicular to the ependymal lining due to their perivenular distribution (Dawson's fingers), NMO lesions are not oval-shaped, located immediately adjacent to the lateral ventricles following the ependymal lining in a disseminated pattern, and are often edematous and heterogeneous [18]. As opposed to what occurs in MS, NMO lesions do not affect the cortical gray matter.

Involvement of the corpus callosum has been described in $18 \%$ of AQP4-seropositive NMO patients. The lesions are multiple, large, and edematous, show heterogeneous signal intensity on T2-weighted images, and sometimes affect the entire thickness of the corpus callosum.

Lesions may also affect areas where AQP4 expression is not particularly high, such as the corticospinal tracts. These lesions, which can be unilateral or bilateral and may affect the posterior limb of the internal capsule and cerebral peduncle of the midbrain, are contiguous and often longitudinally extensive [19].

Other brain MRI findings described in NMO include extensive and confluent hemispheric white-matter lesions and radial hemispheric lesions (sometimes corresponding to an extension of periventricular lesions), which are likely related to vasogenic edema involving the white-matter tracts. These lesions usually do not show mass effect or contrast enhancement, but there may be a "cloud-like" pattern of enhancement, defined as multiple patches of enhancing lesions with blurred margins [18]. In fact, the finding of large hemispheric lesions in a patient suspected of MS should trigger the option of NMO and testing for AQP4 antibodies.

Some of the typical brain MRI findings may be specific to clinical presentations, such as intractable vomiting and hiccup (linear dorsal medullary lesions involving the area postrema and nucleus tractus solitarius), or a syndrome of inappropriate antidiuretic hormone secretion (hypothalamic and periaqueductal lesions) [18].

Distinguishing NMO from MS is critical, particularly in the early stages, since the treatment and prognosis of these disorders differ. In fact, some evidence suggests that MS-modifying treatments such as interferon- $\beta$, natalizumab, and laquinimod exacerbate NMO. By contrast, several immunosuppressants (e.g., azathioprine, rituximab, mitoxantrone) seem to help in preventing NMO relapses.

NMOSD can be associated with systemic autoimmune diseases such as systemic lupus erythematosus and Sjögren's syndrome). Whether the neurologic manifestations are solely due to NMO-spectrum disorder or are a manifestation of these diseases is controversial, although optic neuritis and transverse myelitis are rare presentations of them, and several studies have shown that patients with systemic autoimmune diseases and NMO-IgG-positive antibodies always have optic neuritis, myelitis, or NMO.

\subsubsection{Acute Disseminated Encephalomyelitis (ADEM)}

ADEM is a severe, immune-mediated inflammatory disorder of the CNS that predominantly affects the white matter of the brain and spinal cord. In the absence of specific biologic markers, the diagnosis of ADEM is based on clinical and radiologic features [20]. This disorder affects children more commonly than adults, and, in contrast to MS, shows no gender preponderance. The estimated incidence is 0.8 per 
100,000 population per year. In most cases, the clinical onset of disease is preceded by viral or bacterial infections, usually nonspecific upper-respiratory-tract infections. ADEM may also develop following a vaccination (postimmunization encephalomyelitis). Patients commonly present with nonspecific multifocal symptoms, which developed subacutely over a period of days, frequently associated with encephalopathy (relatively uncommon in MS), defined as an alteration in consciousness (e.g., stupor, lethargy) or a behavioral change unexplained by fever, systemic illness, or postictal symptoms. Although ataxia, encephalopathy, and brainstem symptoms are frequently present in both pediatric and adult cases, certain signs and symptoms appear to be age-related. In childhood ADEM, long-lasting fever and headaches occur more frequently, while in adult cases, motor and sensory deficits predominate. In general, the disease is self-limiting and the prognostic outcome favorable.

Although ADEM usually has a monophasic course, multiphasic forms have been reported, raising diagnostic difficulties in distinguishing these cases from MS. This multiphasic form, which accounts for $<4 \%$ of ADEM cases, is defined as a new encephalopathic event consistent with ADEM, separated by 3 months after the initial illness but not followed by any further events. The second ADEM event can involve either new or re-emergent neurologic symptoms, signs, and MRI findings. Relapsing disease following ADEM that occurs beyond a second encephalopathic event is no longer consistent with multiphasic ADEM but rather indicates a chronic disorder, most often leading to the diagnosis of MS or NMO [21] and should prompt testing for myelin oligodendrocyte glycoprotein (MOG) antibodies, especially in children.

An ADEM event as the first manifestation of the classic relapsing form of MS occurs in 2-10\%. According to the International Pediatric Multiple Sclerosis Study Group, the diagnosis of MS is met if, after the initial ADEM, a second clinical event meets the following three requirements: (1) it is non-encephalopathic; (2) it occurs 3 months or more after the incident neurologic illness; and (3) it is associated with new MRI findings consistent with the McDonald criteria for dissemination in space [21]. The presence of hypointense lesions and two or more periventricular lesions are MRI features that support an MS diagnosis in children with acute CNS demyelination [21]. Unlike the lesions in MS, ADEM lesions are often large, patchy, and poorly marginated on MRI, especially when there are MOG antibodies [22]. There is usually asymmetric involvement of the subcortical and central white matter and cortical gray-white junction of the cerebral hemispheres, cerebellum, brainstem, and spinal cord. Lesions confined to the periventricular white matter and corpus callosum are less common than in MS. The gray matter of the thalamus and basal ganglia is often affected, particularly in children, and typically in a symmetric pattern [23]. However, the frequency of thalamic involvement in adult ADEM does not differ from that of adult MS. This can be explained by the fact that involvement of this structure is less common in adult ADEM than in childhood ADEM. Four patterns of cerebral involvement have been proposed to describe the MRI findings in ADEM [24]: (1) ADEM with small lesions ( $<5 \mathrm{~mm}$ ); (2) ADEM with large, confluent, or tumefactive lesions, and frequent extensive perilesional edema and mass effect; (3) ADEM with additional symmetric deep gray-matter involvement; and (4) acute hemorrhagic encephalomyelitis. Gadolinium enhancement of one or more lesions occurs in $14-30 \%$ of cases [21]. The pattern of enhancement varies and can be complete or incomplete ringshaped, nodular, gyral, or spotty. Although ADEM is usually a monophasic disease, new lesions may be seen on follow-up MRI within the first month of the initial attack.

Most MRI lesions appear early in the course of the disease, supporting the clinical diagnosis of ADEM. Nonetheless, in some cases, there may be a delay of more than 1 month between the onset of symptoms and the appearance of lesions on MRI. Therefore, a normal brain MRI scan obtained within the first days after the onset of neurologic symptoms suggestive of ADEM does not exclude this diagnosis.

The spinal cord is affected in $<30 \%$ of ADEM patients [24], predominantly in the thoracic region. The spinal cord lesion is typically large, causes swelling, and shows variable enhancement. In most ADEM patients, partial or complete resolution of the MRI abnormalities occurs within a few months of treatment. This course is positively associated with a final diagnosis of ADEM.

\subsection{Infectious Inflammatory Demyelinating Disorders}

\subsubsection{Progressive Multifocal Leukoencephalopathy}

Progressive multifocal leukoencephalopathy (PML) is overwhelmingly a disease of the immunocompromised patient and most (55-85\%) cases are related to acquired immunodeficiency syndrome (AIDS). There is a wide age range of involvement, with the peak age of presentation in the sixth decade. The disease is caused by reactivation a papovavirus (JC virus) that selectively attacks the oligodendrocyte, leading to demyelination. Treatment with monoclonal antibody therapy (natalizumab, rituximab, efalizumab) or other immunomodulatory drugs, commonly used in patients with MS and other disorders has also been linked with PML [25]. Untreated patients with PML have an extremely poor prognosis, with death common in the first 6 months following establishment of the diagnosis. Although there is no specific treatment, combination anti-retroviral therapy (cART) has not only resulted in a lower incidence of PML in AIDS patients, but also substantially improved survival times of afflicted patients, now at 50\% 1-year survival [25]. Unfortunately, 
about $20 \%$ of PML cases are linked to a robust inflammatory response to pathogens associated with recovery of the immune system after a period of immunosuppression. This condition, known as PML-IRIS (Immune Reconstitution Inflammatory Syndrome), has been associated with intracranial masses with generous amounts of surrounding vasogenic edema on MRI. Enhancement may also occur [25].

The lesions of PML are characterized by little mass effect or enhancement. Most lesions involve the subcortical white matter and deep cortical layers of the parieto-occipital or frontal white matter, although gray matter and posterior fossa lesions are also common, occurring in up to $50 \%$ of cases. PML lesions tend to be more confluent in their appearance than ADEM lesions and scalloping of the lateral margin of the lesion at the gray matter-white matter junction is common. Subtle signal intensity changes in the white matter may precede clinical suspicion of the PML-IRIS. While development of mass effect and temporary enhancement in the early phase of cART has been linked to better survival, similar imaging manifestations may also be seen in monoclonalantibody treated PML patients [25].

\subsubsection{Human Immunodeficiency Virus Encephalopathy}

Human immunodeficiency virus (HIV) encephalopathy results from direct infection of the brain by the virus itself. Since the advent of cART, the prevalence of the disease has markedly decreased and the temporal progression has been slowed. Most patients are severely immunocompromised at the time of onset and exhibit psychomotor slowing, impaired mental status, and memory difficulties. Histologically, demyelination and vacuolation with axonal loss are noted, along with occasional microglial nodules. Mild cerebral atrophy is the first and sometimes only imaging feature of AIDS dementia complex, also know as HIV dementia, HIV-associated dementia complex, or as HIV-associated neuron-cognitive disorder (HAND). Involvement of the central white matter, basal ganglia, and thalamus is characteristic. Typically, bilaterally symmetric abnormal $\mathrm{T} 2$ hyperintensity in the basal ganglia and small focal areas in the periventricular regions are noted on T2-weighted MR images [26]. Regression of these findings has been seen following institution of cART.

\subsection{White Matter Disease from Toxic Imbalance}

\subsubsection{Chronic Alcohol Ingestion and its Consequences}

Brain abnormalities in alcoholics include atrophy, Marchiafava-Bignami disease, Wernicke encephalopathy, osmotic myelinolysis, and consequences of liver cirrhosis such as hepatic encephalopathy and coagulopathy [27]. All the reported entities are not specific of alcohol and can be found in many other toxic or metabolic conditions. Ethanol direct brain toxicity is caused by under-regulation of receptors of N-methyl-D-aspartate, abnormal catabolism of homocysteine, resulting in an increased susceptibility to glutamate excitatory and toxic effects. Moreover, immune response occurs mediated by lipid peroxidation products that bind to neurons resulting in neurotoxicity. Neuroimaging studies show a characteristic distribution of loss of volume: initially there is infratentorial predominance with atrophy of the cerebellar vermis and hemispheres. Frontal and temporal atrophy is subsequently evident, followed by diffuse atrophy of the brain. The possibility of partial reversibility of these alterations is also observed. In pregnancy, ethanol determines inhibition of maturation of Bergmann's fibers of cerebellum, with consequential marked cerebellar atrophy.

\subsubsection{Hepatic Encephalopathy}

The term hepatic encephalopathy (HE) includes a wide spectrum of neuropsychiatric abnormalities occurring in patients with liver dysfunction. Most cases are associated with cirrhosis and portal hypertension or portal-systemic shunts, but the condition can also be seen in patients with acute liver failure and rarely, those with portal-systemic bypass and no associated intrinsic hepatocellular disease. Although HE is a clinical condition, several neuroimaging techniques, particularly MRI, may eventually be useful for the diagnosis because they can identify and measure the consequences of CNS increase in substances, which, under normal circumstances, are efficiently metabolized by the liver. Classical MR abnormalities in chronic HE include high signal intensity in the globus pallidum on T1-weighted images, likely a reflection of increased tissue concentrations of manganese, and an elevated glutamine/glutamate peak coupled with decreased myo-inositol and choline signals on proton MR spectroscopy, representing disturbances in cell-volume homeostasis secondary to brain hyperammonemia [28]. Recent data have shown that white matter abnormalities, also related to increased CNS ammonia concentration, can also be detected with several MRI techniques: magnetization transfer ratio measurements show significantly low values in otherwise normal-appearing brain white matter, T2-Flair sequences reveal diffuse and focal high signal intensity lesions in the hemispheric white matter, and DWI discloses increased white matter diffusivity. All these MR abnormalities, which return to normal with restoration of liver function, probably reflect the presence of mild diffuse interstitial brain edema, which seems to play an essential role in the pathogenesis of $\mathrm{HE}$.

In acute $\mathrm{HE}$, bilateral symmetric signal-intensity abnormalities on T2-weighted images, often with associated 
restricted diffusion involving the cortical gray matter, are commonly identified. Involvement of the subcortical white matter and the basal ganglia, thalami, and midbrain may also be seen. These abnormalities that can lead to intracranial hypertension and severe brain injury reflect the development of cytotoxic edema secondary to the acute increase of brain hyperammonemia.

\subsubsection{Marchiafava-Bignami Disease}

Marchiafava-Bignami disease is a rare complication of chronic alcoholism, characterized by demyelination and necrosis of the corpus callosum, with rare involvement of extracallosal regions. Etiology remains unknown but is believed to be caused by toxic agents in red wine of low quality and lack of group B vitamins. Rarely, the disease has also been reported in non-alcoholic patients. Symptoms are mainly represented by cognitive deficits, psychosis, hypertonia, and interhemispheric disconnection, until coma and death. Typical MRI features in the acute phase are corpus callosum hyperintensity on T2-weighted sequences and FLAIR, without significant mass effect, with peripheral enhancement. Diffusion is restricted due to cytotoxic edema. In chronic forms, necrosis of the genu and splenium can be detected [29].

\subsubsection{Wernicke Encephalopathy}

Wernicke encephalopathy is an acute condition first described by the French ophthalmologist Gayet in 1875, and later by the German neurologist Wernicke in 1881, caused by a deficiency of B1 (thiamine). It develops frequently but not exclusively in alcoholics. Other potential causes include extended fasting, malabsorption, digitalis poisoning, massive infusion of glucose without B1 in weak patients. The autoptic incidence is reported to be $0.8-2 \%$ in random autopsies, and $20 \%$ in chronic alcoholics. The classic clinical triad of ocular dysfunctions (nystagmus, conjugate gaze palsy, ophthalmoplegia), ataxia, and confusion is observed in only $30 \%$ of cases. Treatment consists of thiamine infusion, and avoids irreversible consequences including Korsakoff dementia or death. Memory impairment and dementia are related to damage of the mammillary bodies, anterior thalamic nuclei and interruption of the diencephalic-hippocampal circuits. Depletion of thiamine leads to failure of conversion of pyruvate to acetyl-CoA and $\alpha$-ketoglutarate to succinate, altered pentose monophosphate shunt, and the lack of Krebs cycle, with cerebral lactic acidosis, intra- and extra-cellular edema, swelling of astrocytes, oligodendrocytes, myelin fibers, and neuronal dendrites. Neuropathological aspects include neuronal degeneration, demyelination, hemorrhagic petechiae, proliferation of capillaries and astrocytes in peri-aqueductal gray substance, mammillary bodies, thalami, pulvinar, III cranial nerves nuclei, and cerebellum. On MRI, bilateral and symmetrical hyperintensities on T2-weighted sequences and FLAIR are evident at the level of the already mentioned structures, mainly mammillary bodies and thalami [30, 31]. Rarely, cortex of the forebrain can be involved. DWI shows areas of reduced apparent diffusion coefficient (ADC) due to cytotoxic edema, although the ADC can sometimes be high due to the presence of vasogenic component. Rarely, T1-weighted images show bleeding ecchymotic hemorrhages in the thalami and mammillary bodies, a sign considered clinically unfavorable. In $50 \%$ of cases, contrast enhancement is present in periaqueductal regions. Marked contrast enhancement of mammillary bodies is evident in $80 \%$ of cases, even prior to the development of visible changes in T2-weighted sequences and is considered highly specific for WE. In chronic forms, change of signal in T2-weighted sequences becomes less prominent due to the diffuse brain atrophy, more pronounced at the level of mesencephalon and mammillary bodies.

\subsubsection{Osmotic Demyelination Syndrome}

Osmotic demyelination syndrome (ODS) usually occurs in the setting of osmotic changes, typically with the rapid correction of hyponatremia. This causes destruction of the blood-brain barrier with hypertonic fluid accumulation in extracellular space, resulting in a non-inflammatory demyelination. The most common damage is in the pontine fibers [32]. ODS is observed in alcoholics with nutritional deficiency. The most common symptoms include paralysis, dysphagia, dysarthria, and pseudobulbar palsy. Death is frequent. Rarely, ODS affects other regions, especially basal ganglia, thalami, deep white matter. MRI usually shows an area of high signal on T2-weighted sequences in the central part of the pons (central pontine myelinolysis), sparing ventro-lateral portions and corticospinal tracts. The lesion is moderately hypointense in $\mathrm{T} 1$ and may show positive contrast enhancement. If the patient survives, the acute phase can evolve into a cavitated pontine lesion.

\subsection{White Matter Disease Associated with Radiation Therapy and Chemotherapy}

Treatment strategies designed to target cancer cells are commonly associated with deleterious effects to multiple organ systems, including the CNS. As both radiation and chemotherapy alone can be associated with significant toxicity, the 
combination of radiation and chemotherapy may be particularly harmful to the CNS. With advanced treatment regimens and prolonged survival, neurological complications are likely to be observed with increasing frequency.

Neurotoxicity can result from direct toxic effects of the drug or radiation on the cells of the CNS, or indirectly through metabolic abnormalities, inflammatory processes, or vascular adverse effects.

Recognition of treatment-related neurologic complications is critically important, because symptoms may be confused with metastatic disease, tumor progression, paraneoplastic disorders, or opportunistic infections, and discontinuation of the offending drug may prevent irreversible CNS injury.

\subsubsection{Radiation Injury and Necrosis}

It is widely accepted that the white matter of the CNS is prone to radiation-induced injury, compared with gray matter. Radiation encephalopathy has been classically divided into three stages according to its timing after radiotherapy: early, early-delayed, and late-delayed reactions [33]. Within the first several weeks of therapy, patients may experience acute declines with focal neurologic deficits. These effects are possibly related to increased edema, which has been supported by the observation that steroid treatment often results in clinical improvement. Early-delayed adverse effects usually occur within 1-6 months of treatment and are thought to be a result of demyelination. This syndrome is characterized by somnolence, fatigue, and cognitive dysfunction, consistent with dysfunction of the frontal network systems. Late-delayed side effects occur months to years after cessation of treatment and are largely irreversible and progressive. These late changes are commonly associated with progressive cognitive deficits. In more severe cases of late-delayed radiation injury, imaging and histopathological studies may demonstrate leukoencephalopathy and/or focal necrosis.

In all types of white matter radiation-induced damage, CT and mainly MRI may demonstrate variable degrees of white matter signal changes related to an increase in free tissue water in the involved areas. This may result from endothelial damage, causing increased capillary permeability and vasogenic edema, or from demyelination. However, the degree of these white matter changes correlates poorly with the functional deterioration observed in the patients. MRI findings in early reactions occurring during course of treatment are nonspecific. MRI may be normal or demonstrate poorly defined multifocal lesions in both hemispheres that usually disappear spontaneously. MRI in early-delayed reactions also may show signal changes involving not only the hemispheric white matter but also the basal ganglia and the cerebral peduncles, which resolve completely without treatment. These early-delayed changes have been reported in children with acute lymphatic leukemia who have been treated with both whole brain irradiation and chemotherapy. These changes have no correlation with clinical manifestations and have no clear prognostic significance. Late-delayed reactions can be subdivided into diffuse and focal radiation necrosis injury.

Diffuse radiation injury is characterized by white matter changes that are "geographic" in nature, i.e., the areas of abnormal signal intensity or attenuation are limited to the regions of the brain that conform to the radiation portal. This can produce striking differences between the involved zones and the spared surrounding white matter. The involved territories are often symmetric and do not enhance on post-contrast studies. While originally reported in children with leukemia, diffuse necrotizing leukoencephalopathy has also been observed following treatment for many other malignancies in both children and adults. The disease may occur following chemotherapy alone, but the incidence of disease is highest when chemotherapy is combined with radiation therapy. Both the histologic findings and imaging features bear resemblance to radiation necrosis. Axonal swelling, demyelination, coagulation necrosis, and gliosis dominate the histologic picture.

Diffuse white matter changes, with hypoattenuation on $\mathrm{CT}$ and $\mathrm{T} 1$ and $\mathrm{T} 2$ prolongation on MRI, are common and often involve an entire hemisphere. Microbleeds can occur as a sign of vasculopathy. Radiation-induced leukoencephalopathy may be associated with progressive brain atrophy, and patients may present with cognitive decline, gait abnormalities, and urinary incontinence. However, the more common mild-to-moderate cognitive impairment is inconsistently associated with radiological findings, and frequently occurs in patients with normal-appearing scans. Recently, more sensitive tools to quantify the damage to brain tissue, such as diffusion tensor imaging (DTI) have been applied in patients treated with brain radiotherapy and have shown early and progressive diffuse changes in normal-appearing white matter indicating radiationinduced demyelination and mild structural degradation of axonal fibers, which are undetectable with conventional MRI.

Focal radiation necrosis usually manifests as a ring-like or irregular enhancing mass lesion located in the white matter, which may become hemorrhagic. The MRI features commonly seen in radiation necrosis include a soap bubblelike interior and Swiss cheese-like interior. Compared with lesions with the soap bubble pattern, Swiss cheese lesions are larger, more variable in size, and more diffuse. This pattern can be visualized as a result of diffuse necrosis affecting the white matter and cortex with diffuse enhancement at the 
margins with intermixed foci of necrosis. The rim of enhancement is often thinner, more uniform, and more aligned to the gray matter-white matter junction than in malignant tumors. As radiation necrosis progresses, it can lead to severe shrinkage of the white matter and cortex and result in focal brain atrophy with hydrocephalus.

Quite frequently is impossible to distinguish radiation necrosis from recurrent malignant brain tumor, such as glioblastoma multiforme, using conventional MRI. Metabolic imaging (e.g., positron emission tomography) may facilitate differentiating between the two diseases as radiation necrosis is iso-to-hypometabolic while recurrent high-grade tumors are typically hypermetabolic. MR spectroscopy (MRS) may also be useful as radiation necrosis frequently shows a characteristic lactic acid peak and near-normal peaks for $\mathrm{N}$-acetylaspartate (NAA) and choline while recurrent high-grade gliomas typically show elevated choline levels compared to NAA without or with elevated lactic acid levels. Perfusion imaging can identify the areas of increased blood flow associated with tumor recurrence, whereas radiation necrosis is not expected to contain any increased blood flow [34].

\subsubsection{Chemotherapy-Associated Neurotoxicity}

Neurotoxicity has been observed with virtually all categories of chemotherapeutic agents [35]. Neurologic complications may range from acute encephalopathy, headache, seizures, visual loss, cerebellar toxicity, and stroke to chronic side effects, including chronic encephalopathy, cognitive decline, and dementia.

Among the most puzzling aspects of cancer therapyrelated toxicity is the occurrence of delayed and progressive neurological decline, even after cessation of treatment. Anticancer agents affect brain function through both direct and indirect pathways. It is also conceivable that additional variables play important roles, including the timing of treatment, combination of different treatment modalities, patient age, integrity of the blood-brain barrier, and cognitive function prior to treatment initiation.

Imaging studies have provided evidence that structural and functional CNS changes occur in a significant number of patients treated with chemotherapy. Some agents, such as methotrexate or carmustine, are well known to cause a leukoencephalopathy syndrome, especially when administered at a high dose, intrathecally, or in combination with cranial radiotherapy. Nonenhancing, confluent, periventricular white matter lesions, necrosis, ventriculomegaly, and cortical atrophy characterize this syndrome. White matter abnormalities following high-dose chemotherapy have been detected in up to $70 \%$ of treated individuals and usually have a delayed onset of several months.
A delayed leukoencephalopathy syndrome with distinct DWI abnormalities on MRI indicative of cytotoxic edema within cerebral white matter has been recently described. This syndrome appeared to mimic a stroke-like syndrome and has seen mainly in patients receiving methotrexate, 5-fluorouracil (5-FU), carmofur, and capecitabine.

\subsection{Vascular Causes of White Matter Disease}

\subsubsection{Posterior Reversible Encephalopathy Syndrome (PRES)}

Although not a truly demyelinating condition, reversible encephalopathy syndrome is noteworthy because of its affinity for the posterior cerebral white matter territories and is important in the differential diagnosis of demyelinating disease. Under normal circumstances, cerebral perfusion pressure is maintained at a relatively constant level by autoregulation, a physiologic mechanism that compensates for wide changes in systemic blood pressure. Hypertensive encephalopathy is believed to result from loss of normal autoregulation, with competing regions of vasodilatation and vasoconstriction, and endothelial dysfunction. The vessels of the posterior cerebral circulation, lacking less sympathetic innervation compared to those of the anterior circulation, are unable to vasoconstrict in a normal manner and bear the brunt of these vascular changes. Reversible vasogenic edema is the result and is associated with visual field deficits, as well as headaches, somnolence, and an overall impaired mental status. The terms posterior reversible encephalopathy syndrome (PRES) and reversible posterior leukoencephalopathy syndrome (RPLS) have been popularized in the literature to describe this scenario that is most commonly seen in hypertensive states and/or the presence of immunosuppression (particularly cyclosporine A and tacrolimus), chemotherapy, eclampsia, and renal failure. While it commonly involves the posterior cerebral white matter, other sites may also be affected including unilateral cerebral hemispheric or isolated brainstem involvement in patients following aortic valve surgery [36, 37]. Accordingly, it has been suggested that perhaps the terminology should be changed to simply "reversible encephalopathy" [38].

On MR studies, bilaterally symmetric abnormal T2 hyperintensity, representing vasogenic edema, is most commonly seen in the distribution of the posterior circulation, although other sites including the frontal lobes and corpus callosum may be noted as well. Cortical and subcortical lesions may be better detected on FLAIR sequences. DWI may be normal or show restricted water diffusion in regions of infarction that correlate with poorer prognosis [23]. Susceptibilityweighted imaging may show areas of hemorrhage within 
involved territories. With early treatment and limited involvement of the brain, many of these imaging abnormalities will completely resolve and most patients recover within 2 weeks [39]. However, when larger areas or regions of infarction are involved, permanent neurologic deficits or even death are possible. Vascular narrowing has been observed on angiographic studies. Perfusion studies reported in the literature indicate normal to increased perfusion in these zones. When biopsies of these regions have been performed, white matter edema is seen histologically.

\subsubsection{Aging and Ischemic Demyelinating Disorders}

Small focal lesions on T2-weighted images are quite common in the white matter of adult subjects [40]. They are not associated with mass effect, do not enhance, and are typically isointense compared to normal white matter on T1-weighted images. When these lesions have been biopsied, histologic examination reveals a spectrum of findings including gliosis, (partial) loss of myelination, and vasculopathy. They tend to be located in the deep white matter of the centrum semiovale. In contrast to MS, lesions do not involve the corpus callosum or the juxtacortical U-fibers, important distinguishing features [3]. Since the lesions are so ubiquitous and appear to be a part of "normal" aging, various terms have been proposed: senescent white matter changes or disease, deep white matter ischemia, leukoaraïosis, etc. In general, the more lesions present, the more likely it is that the patient will have cognitive problems or difficulties with neuropsychological testing. However, it is not possible to predict a particular patient's status simply based on the imaging appearance alone.

In adult patients between 30 and 50 years of age, the presence of periventricular and subcortical lesions in a patient with a family history of similarly affected relatives should raise the possibility of cerebral autosomal dominant arteriopathy with subcortical infarcts and leukoencephalopathy (CADASIL). A defect in the notch3 gene on the long arm of chromosome 19 has been identified and apparently evokes an angiopathy affecting small and medium-sized vessels. Most lesions occur in the frontal and temporal lobes and less commonly in the thalamus, basal ganglia, internal and external capsules, and brain stem [41].

\subsection{Conclusion}

MRI of the brain and spinal cord is of vital importance in the diagnosis of MS, the most important idiopathic inflammatory CNS disorder [42]. Dissemination in space and time on MRI is not limited to MS and can occur in NMO, and spinal cord imaging is important in the differential diagnosis.
Increasingly, MRI is used to monitor MS treatment and their complications such as PML.

\section{Take Home Message}

- MRI of the brain and the spinal cord should be performed in any patient suspected of MS to show dissemination in space according to McDonald criteria. Additional gadolinium enhancement or new T2 lesions demonstrate dissemination in time and allow a final diagnosis and treatment initiation. Spinal cord MRI with multiple short segment lesions is almost pathognomonic for MS and a negative cord scan is a red flag in MS.

\section{References}

1. Smith AB, Smirniotopoulos JG. Imaging evaluation of demyelinating processes of the central nervous system. Postgrad Med J. 2010;86:218-29.

2. Cañellas AR, Gols AR, Izquierdo JR, et al. Idiopathic inflammatory-demyelinating diseases of the central nervous system. Neuroradiology. 2007;49:393-409.

3. Aliaga ES, Barkhof F. MRI mimics of multiple sclerosis. Handb Clin Neurol. 2014;122:291-316.

4. Rovira À, Barkhof F. Multiple sclerosis and variants. In: Barkhof F, Jager R, Thurnher M, Rovira Cañellas A, editors. Clinical neuroradiology. Cham: Springer; 2018.

5. Bagnato F, Jeffries N, Richert ND, et al. Evolution of T1 black holes in patients with multiple sclerosis imaged monthly for 4 years. Brain. 2003;126:1782-9.

6. Ciccarelli O, Cohen JA, Reingold SC, Weinshenker BG. Spinal cord involvement in multiple sclerosis and neuromyelitis optica spectrum disorders. Lancet Neurol. 2019;18(2):185-97.

7. Cotton F, Weiner HL, Jolesz FA, et al. MRI contrast uptake in new lesions in relapsing-remitting MS followed at weekly intervals. Neurology. 2003;60:640-6.

8. Masdeu JC, Quinto C, Olivera C, et al. Open-ring imaging sign: highly specific for atypical brain demyelination. Neurology. 2000;54:1427-33.

9. Popescu BF, Lucchinetti CF. Pathology of demyelinating diseases. Annu Rev Pathol. 2012;7:185-217.

10. Stadelmann C, Ludwin S, Tabira T, et al. Tissue preconditioning may explain concentric lesions in Baló's type of multiple sclerosis. Brain. 2005;128:979-87.

11. Hardy TA, Miller DH. Baló's concentric sclerosis. Lancet Neurol. 2014;13:740-6.

12. Wuerfel J, Rovira A, Paul F, Barkhof F. Neuromyelitis optica spectrum disorders (NMOSD). In: Barkhof F, Jager R, Thurnher M, Rovira Cañellas A, editors. Clinical neuroradiology. Cham: Springer; 2019.

13. Trebst C, Jarius S, Berthele A, et al. Update on the diagnosis and treatment of neuromyelitis optica: recommendations of the Neuromyelitis Optica Study Group (NEMOS). J Neurol. 2014;261:1-16.

14. Lennon VA, Wingerchuk DM, Kryzer TJ, et al. A serum autoantibody marker of neuromyelitis optica: distinction from multiple sclerosis. Lancet. 2004;364:2106-12.

15. Wingerchuk DM, Lennon VA, Pittock SJ, et al. Revised diagnostic criteria for neuromyelitis optica. Neurology. 2006;66:1485-9. 
16. Wingerchuk DM, Banwell B, Bennett JL, et al. International consensus diagnostic criteria for neuromyelitis optica spectrum disorders. Neurology. 2015;85:177-89.

17. Yonezu T, Ito S, Mori M, et al. "Bright spotty lesions" on spinal magnetic resonance imaging differentiate neuromyelitis optica from multiple sclerosis. Mult Scler. 2014;20:331-7.

18. Misu T, Fujihara K, Nakashima I, et al. Intractable hiccup and nausea with periaqueductal lesions in neuromyelitis optica. Neurology. 2005;65:1479-82.

19. Kim HJ, Paul F, Lana-Peixoto MA, et al. MRI characteristics of neuromyelitis optica spectrum disorder: an international update. Neurology. 2015;84:1165-73.

20. Auger C, Rovira A. Acute disseminated encephalomyelitis and other acute parainfectious syndromes. In: Barkhof $\mathrm{F}$, Jager R, Thurnher M, Rovira CA, editors. Clinical neuroradiology. Cham: Springer; 2018.

21. Verhey LH, Branson HM, Shroff MM, et al. MRI parameters for prediction of multiple sclerosis diagnosis in children with acute CNS demyelination: a prospective national cohort study. Lancet Neurol. 2011;10:1065-73.

22. Hacohen Y, Mankad K, Chong WK, et al. Diagnostic algorithm for relapsing acquired demyelinating syndromes in children. Neurology. 2017;89:269-78.

23. Covarrubias D, Luetmer P, Campeau N. Posterior reversible encephalopathy syndrome: prognostic utility of quantitative diffusionweighted MR images. AJNR Am J Neuroradiol. 2002;23:1038-48.

24. Tan CS, Koralnik IJ. Progressive multifocal leukoencephalopathy and other disorders caused by JC virus: clinical features and pathogenesis. Lancet Neurol. 2010;9:425-37.

25. Wattjes MP, Barkhof F. Diagnosis of natalizumab-associated progressive multifocal leukoencephalopathy using MRI. Curr Opin Neurol. 2014;27:260-70.

26. Gottumukkala RV, Romero JM, Riascos RF, et al. Imaging of the brain in patients with human immunodeficiency virus infection. Top Magn Reson Imaging. 2014;23:275-91.

27. Geibprasert S, Gallucci M, Krings T. Alcohol-induced changes in the brain as assessed by MRI and CT. Eur Radiol. 2010;20:1492-501.

28. Rovira A, Alonso J, Cordoba J. MR imaging findings in hepatic encephalopathy. AJNR Am J Neuroradiol. 2008;29:1612-21.

29. Arbelaez A, Pajon A, Castillo M. Acute Marchiafava-Bignami disease: MR findings in two patients. AJNR Am J Neuroradiol. 2003;24:1955-7.
30. Gallucci M, Bozzao A, Splendiani A, et al. Wernicke encephalopathy: MR findings in five patients. AJR Am J Roentgenol. 1990;155:1309-14.

31. Zuccoli G, Santa Cruz D, Bertolini M, et al. MR imaging findings in 56 patients with Wernicke encephalopathy: nonalcoholics may differ from alcoholics. AJNR Am J Neuroradiol. 2009;30:171-6.

32. Ruzek KA, Campeau NG, Miller GM. Early diagnosis of central pontine myelinolysis with diffusion-weighted imaging. AJNR Am J Neuroradiol. 2004;25:210-3.

33. Soussain C, Ricard D, Fike JR, et al. CNS complications of radiotherapy and chemotherapy. Lancet. 2009;374:1639-51.

34. Baehring JM, Fulbright RK. Delayed leukoencephalopathy with stroke-like presentation in chemotherapy recipients. J Neurol Neurosurg Psychiatry. 2008;79:535-9.

35. Godi C, Falini A. Radiological findings of drug-induced neurotoxic disorders. In: Barkhof F, Jager R, Thurnher M, Rovira Cañellas A, editors. Clinical neuroradiology. Cham: Springer; 2019.

36. Wijdicks EF, Campeau N, Sundt T. Reversible unilateral brain edema presenting with major neurologic deficit after valve repair. Ann Thorac Surg. 2008;86:634-7.

37. McKinney AM, Short J, Truwit CL, et al. Posterior reversible encephalopathy syndrome: incidence of atypical regions of involvement and imaging findings. AJR Am J Roentgenol. 2007;189:904-12.

38. Casey SO, Sampaio RC, Michel E, Truwit CL. Posterior reversible encephalopathy syndrome: utility of fluid-attenuated inversion recovery MR imaging in the detection of cortical and subcortical lesions. AJNR Am J Neuroradiol. 2000;21:1199-206.

39. Post JD, Beauchamp NJ. Reversible intracerebral pathologic entities mediated by vascular autoregulatory dysfunction. Radiographics. 1998;18:353-67.

40. Kloppenborg RP, Nederkoorn PJ, Geerlings MI, van den Berg E. Presence and progression of white matter hyperintensities and cognition: a meta-analysis. Neurology. 2014;82:2127-38.

41. van dem Boom R, Lesnick Oberstein S, et al. Cerebral autosomal dominant arteriopathy with subcortical infarcts and leukoencephalopathy: MR imaging changes and apolipoprotein E genotype. AJNR Am J Neuroradiol. 2006;27:359-62.

42. Rovira À, Wattjes MP, Tintoré M, et al. Evidence-based guidelines: MAGNIMS consensus guidelines on the use of MRI in multiple sclerosis-clinical implementation in the diagnostic process. Nat Rev Neurol. 2015;11:471-82.

Open Access This chapter is licensed under the terms of the Creative Commons Attribution 4.0 International License (http://creativecommons. org/licenses/by/4.0/), which permits use, sharing, adaptation, distribution and reproduction in any medium or format, as long as you give appropriate credit to the original author(s) and the source, provide a link to the Creative Commons license and indicate if changes were made.

The images or other third party material in this chapter are included in the chapter's Creative Commons license, unless indicated otherwise in a credit line to the material. If material is not included in the chapter's Creative Commons license and your intended use is not permitted by statutory regulation or exceeds the permitted use, you will need to obtain permission directly from the copyright holder. 